\title{
IT'S NEVER TO LATE FOR ERAS PATHWAYS: ESP BLOCK AT T7 FOR LAPAROTOMIC COLONIC RESECTION IN A 92 YEARS OLD WOMAN
}

F. Costa1, G. Pascarella1, R. Del Buono², A. Strumia1, E. Barbara², F.E. Agrò1 ${ }^{1}$ Campus Bio Medico Unversity Hospital, Unit of Anesthesia- Intensive Care and Pain Management, Rome, Italy. 2Humanitas Mater Domini Hospital, Anesthesia, Castellanza Varese, Italy.

Background and Aims: In ERAS programs pain and PONV are the worst enemies, as they compromise early mobilization and nutrition. Pain control with multimodal strategy is mandatory to reduce opioids intake that could lead to PONV. Regional anesthesia plays a significant role in opioid sparing management. Aged people may have even greater benefit from those pathways [1]. We describe a case of a 92 years old woman scheduled for laparoscopic left colonic resection under general anesthesia and bilateral ESP block at T7.

FIGURE LEGEND:

Tm - Trapezius muscle Rm - Rhomboid muscle ESm - Erector Spinae muscles

LA - local anesthetic longitudinal spread far from needle tip

Green Arrows - needle path

Red Arrows - 7th rib localization at the lateral inferior border of rhomboid muscle, to easily find $\mathrm{T} 7$ transverse process.

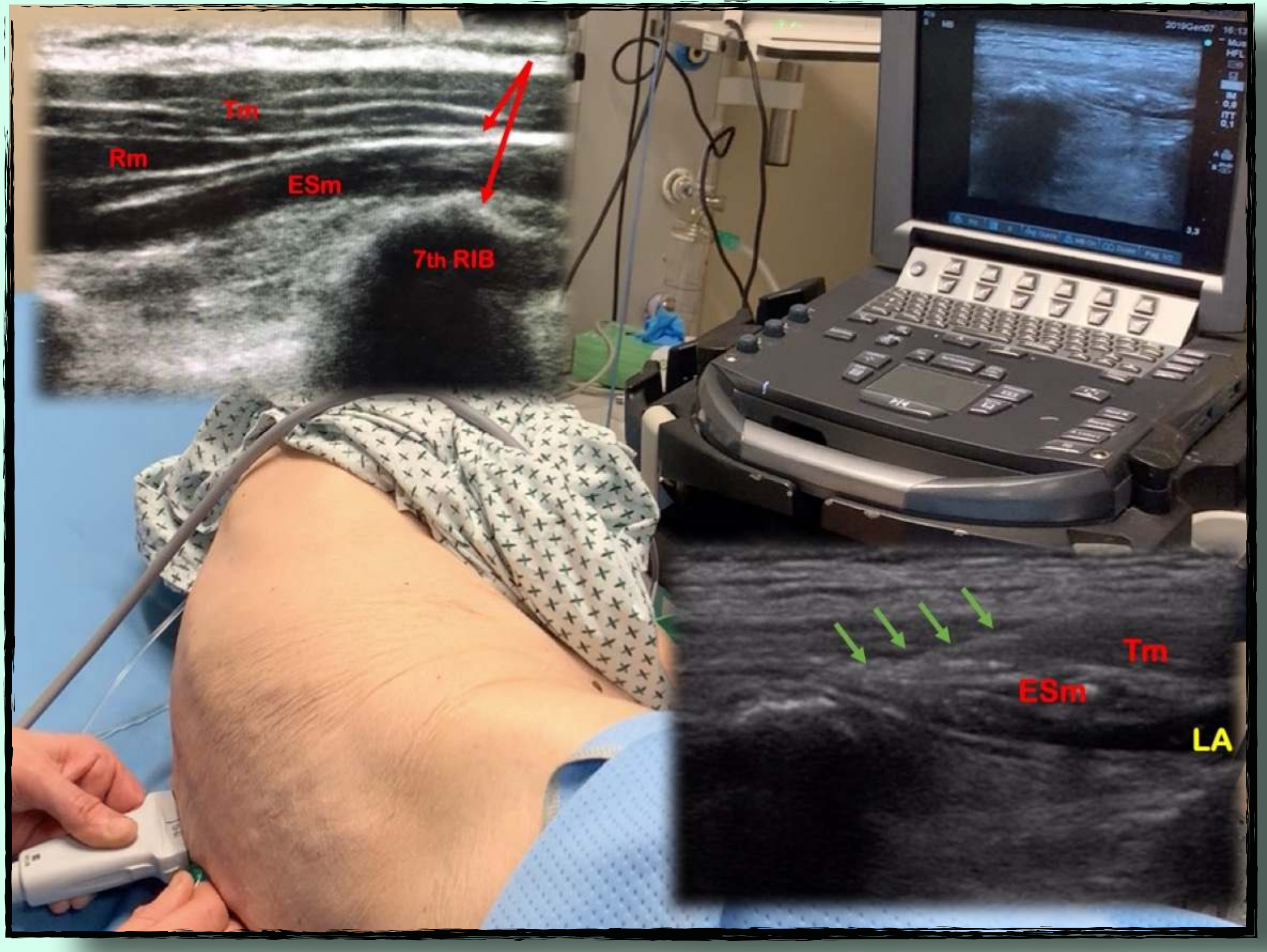

Methods: Informed consent was obtained. In lateral position, T7 transverse processes were identified (fig.1), ESP block was bilaterally performed; 1 to $2 \mathrm{ml}$ of inactive fluid were injected to verify the correct tip position, then $20 \mathrm{ml}$ of a local anesthetic mixture of $2 \%$ mepivacaine and $0,75 \%$ ropivacaine were injected in each side. A light, total intravenous general anesthesia was administered. Because of visceral adhesions, the procedure was converted to laparotomic approach. Despite this, after induction dose of fentanyl, $(100 \mathrm{mcg})$ intraoperative opioids were no longer required.

Results: Postoperatively, the patient only complained bladder catheter discomfort, thus it was promptly removed. No pain scores above NRS 3, nor PONV were registered. Analgesia was maintained with paracetamol and ketorolac. Oral intake was tolerated within 24 hours. She was discharged home on POD3.

Conclusions: ESP block seems to be an effective technique for the entire abdominal wall analgesia. $[2,3]$ We consider it a promising technique in ERAS programs for colorectal surgery. Advanced age [1] and type of surgery approach are not a limitation.

\section{References:}

1. O. Ljungqvist, M Hubner Enhanced recovery after surgery - ERAS - principles, practice and feasibility in the elderly Aging Clinical and Experimental Research. 2018; 30:249-252

2. María Bermúdez López, et Al Erector Spinae Block. A narrative review Central European Journal of Clinical Research. 2018;1(1):28-39

3. Chin KJ, Adhikary S, Sarwani N, Forero M. The analgesic efficacy of pre-operative bilateral erector spinae plane (ESP) blocks in patients having ventral hernia repair. Anaesthesia 2017;7(4):452-60

Contact Details: Dr. Fabio Costa Md - Email: F.Costa@Unicampus.It - Cell. +39-3356526433 